\title{
The Application Research of Improved Bat Algorithm for Time Table Problem \\ Wenxiang Liu ${ }^{1, a}$ \\ ${ }^{1}$ Gansu Normal University for Nationalities, Hezuo, gansu, 747000, China \\ apwjupeng@163.com
}

Keywords: Bat Algorithm; Time table problem; active evolutionary operator; invalid bat

\begin{abstract}
The time table problem is a typical NP complete problem, when the bat algorithm solves this problem; it will quickly converge, and can get the local optimal solution but not the global optimal solution. Therefore, we use the advantage of active evolutionary operator to improve the bat algorithm, and to solve TTP problem. We do a lot of experiments and evaluate the performance of the improved bat algorithm. The experiment results show that improved bat algorithm is a more superior algorithm to apply to the TTP problem.
\end{abstract}

\section{Introduction}

In the last few years, colleges and universities around the world are expanding enrollment in order to develop more high-quality personnel, various countries' educational reform is also thorough unceasingly ${ }^{[1-3]}$. The essence of course scheduling question is the curriculum, the teacher and the student should be assigned in the appropriate time section to the appropriate classroom. It is a multi-objective scheduling problem which involve more factors, and known as the Time table Problem in operations research (Time table Problem, called TTP). Automated Course Scheduling algorithm, TTP, was proved to be NP-complete problem as early as in the 70s. The computing time is exponential increase, and integration time and space as dual constraints. From classroom (space) point of view it is similar to the bin-packing problem. But increased many restraints in the time. So it is a more complex problem than the bin-packing problem. We can establish theoretical profundity from this thesis. At present, in mathematics did not have an general algorithm to be able to solve the NP complete problem well. Solving the NP complete problem only to be able to depend on the approximate method, many scholars have conducted the research regarding this. In this thesis we will discuss and evaluate the performance under the unified test platform to the commonly used TTP algorithm ${ }^{[4-5]}$.

\section{Arrangement of conditions to be followed}

In the Course Scheduling question, it is Involves 5 restricted mutually factors, the class and grade, the teacher, the time, the curriculum, the classroom. The schedule question's solution process is to any class and grade, the teacher, the curriculum, the classroom arranges same idle time. When arrangement cannot have the conflict, simultaneously satisfies the experience and general knowledge as far as possible.

We will restrain divides into two levels constraints; first-level restraint for the basic restraint which must satisfy the second-level restraint on the trimming in the first-level restraint satisfied foundation to better meet the requirements.

First-level restraint: each curriculum serial number must assign in one classroom, attends class or the instruction by one teacher; several curriculum per week must have a certain time intervals; The identical teacher cannot in the same time in the different classroom on different subjects; Within the same classroom at the same time can not be arranged two or more courses; Classroom seat quantity must be greater than or equal to the students; Some classes must use the special classroom or multimedia and successive periods, such as art design; The identical class's students cannot have 
two or two more courses in the same time. These are the conditions that must be met when arranging, otherwise is unable to carry on the normal teaching.

Second-level restraint: the teacher's special request, some teachers want the lecture time to be concentrated; Some teachers do not want to class in the evening.

\section{Bat algorithm}

The bat algorithm $(\mathrm{BA})^{[6]}$ is a new heuristic algorithm ${ }^{[9]}$, it is proposed by XinShe Yang in 2010. Yang illustrates the basic idea of this algorithm and puts forward the basic assumption of the bat algorithm:

1) All bats use their echolocation perception and the distance of the target, they identify the target and the different of the background obstacles in a mysterious way.

2) The location of bats are $x_{i}$, flying with the speed of $v_{i}$, they search target at a fixed frequency $f$, variable wavelength $\lambda$ and loudness $A_{i}$. They can determine the distance between himself and the prey and adjust the pulse wavelength (frequency) automatically, at the same time, when they close to the target, they adjust the pulse frequency $f_{i} \in[0,1]$.

3 ) There are a lot of changes in loudness, the assumption it is from the maximum value of $A_{\max }$ changes to the fixed minimum value of $A_{\text {min }}$.

Since the algorithm is proposed, some scholars have applied this algorithm to solve continuous function optimization problems, and have achieved good results. This paper presents a novel bat algorithm to solve the time table problem, the simulation results show that the algorithm has better convergence speed and precision, and it can effectively solve the time table problem.

\section{The novel bat algorithm for Scheduling System}

Traditional bat algorithm has the shortcomings of early convergence and variation problem, makes the improvement based on the bat algorithm. We can use mutation factor to improve early convergence and mutation question. And combine the novel bat algorithm to find the minimum value in the optimization problem ${ }^{[7]}$.

Composite each course and teacher, to solve "multi-class", "one more class for one teacher "conflict, fixed time interval problems. We use the mixed style teacher to code, set the teachers' hybrid coding as the mutation factors. The mutation factors are composed by the teacher number, the class and grade serial number, the curriculum number, the curriculum characteristic. As shown in Fig.1.

\begin{tabular}{|l|l|l|l|l|l|l|}
\hline Class 1 & $\longrightarrow$ & Monday 1,2 & Monday 3,4 & $\cdots \cdots \cdots \cdots$ & Friday 7,8 \\
\hline Class 2 & Teacher & & & & \\
\hline Class 3 & Course & & & & \\
\hline Classroom & & & & & \\
\hline
\end{tabular}

Fig. 1. Mutation factors encoding

There are two steps for initial population's production: The first step, for each class $C_{i}(i=1 \ldots$ $n)$, teacher numbers will be arranged randomly in 20 time slices. If there are 5 classes $(i=5)$, then we can get chromosome after five circulations, according to the population scale's size which assigned produces the certain amount individual to form the initial population. Of course, there are possible initial generation of individuals, but also feasible. The second step, use novel bat algorithm to find the near-optimum solution from the feasible solution. This step can be described as: through the loop, the fitness of a feasible solution to a given fitness value, that solution is the optimal solution obtained; After massively, if the feasible solution sufficiency value cannot achieve the sufficiency value which assigns, then the conflict will take the penalty function, and define the penalty function in the sufficiency function. 
The novel bat algorithm selects next generation population in the evolution by basing on the fitness value of each individual. Fitness function set directly influence on speed of the novel bat algorithm convergence and to find optimal solutions. In this article, the fitness function's design concept is weight sum conflict type which exists in each chromosome, If one of chromosomes violate rule $i$, its value will be set to $1 P_{i}$, the conflict which exists to the chromosome carries on the weighting to sum and to add on 1, then make reciprocal, the chromosome sufficiency function value is bigger, then indicate that has the good teaching time interval and the classroom, its next-generation evolution of the probability of survival is larger.

Selecting operation is used to simulate the phenomenon of natural selection in biosphere. It selects a high fitness chromosome from the old population, and put into the matching set, and prepare to chromosome crossover and mutation operation to generate a new population. Higher fitness chromosomes are selected more possibly. We choose a method of local selection method, its truncation selection method.

In truncation selection method, chromosome is sorted by fitness function value from high to low. Only the best individual can be selected as parent individual. The percentage parameters, which is used to determine the parent individual, is called truncation threshold, its range is $10 \% \sim 50 \%$, the individual, which out of threshold, can not produce sub-individuals.

The process of improved bat algorithm:

Step1: Parameters initialization. Parameters, which need to be initialized, include: the population size $N$, the maximum algebra $M$, the selection rate $P_{s}$, and the sequence $T_{k l}, T_{k 2}, T_{k 3}, T_{k 4}$.

Step2: Generate an initial population of population size $N$ randomly according to the encoding scheme.

Step3: Begin to iterate.

Step4: Do the chaotic crossover operation to the chromosomes in population according to the current value of $T_{k l}$ and $T_{k 2}$. All the chromosomes, generated by mixed crossover operation, constitute a sub-population.

Step5: Do the mutation operation to the chromosomes in the sub-populations according to the current value of $T_{k 3}$ and $T_{k 4}$.

Step6: Calculate the fitness of all chromosomes in populations and sub-populations.

Step7: Do the selection operation to all of the chromosomes in populations and sub-populations according to the selection probability $P_{s}$, eliminate low adaptation chromosomes, the else chromosomes retained constitute new populations.

Step8: When the standard of iteration stopping is $m$, end the iteration, output current optimal chromosome. It is the chromosome of highest adaptation degree, the optimal chromosome is the original problem better solution; otherwise returns step 4.

\section{The analysis of simulation}

In test, we choose to two periods each morning and a time period each afternoon, fifteen time period in a week; we choose two school buildings classrooms.

Set population size is 50 , generation is 50 , meta course is 113 , Test group 1 and test group 2 set genetic generation to be 100 and 50 .

Test One: Establishing populations time is $3.775 \mathrm{~s}$, failure is 2, time is $11.466 \mathrm{~s}$.

Table 1. time consumption of algorithm

\begin{tabular}{|c|c|c|c|c|}
\hline Algorithm name & Population size & generation & time & Feature value \\
\hline $\begin{array}{c}\text { Simulated } \\
\text { Annealing }\end{array}$ & 50 & 50 & $10.86 \mathrm{~s}$ & 209 \\
\hline Bat algorithm & 50 & 50 & $7.76 \mathrm{~s}$ & 206 \\
\hline $\begin{array}{c}\text { Novel bat } \\
\text { algorithm }\end{array}$ & 50 & 50 & $8.002 \mathrm{~s}$ & 245 \\
\hline
\end{tabular}

The table shows that bat algorithms time-consuming is least under the same conditions, novel bat algorithm is slightly more, because the search use part of time, but the characteristic value is greatly 
improved. Once again, we will compare the three algorithms, prerequisite is that adaptive parameters are same, test their number of iterations, the results are as follows:

Table 2. the number of test iterations

\begin{tabular}{|c|c|c|c|}
\hline Adaptive parameter & Simulated Annealing & Bat algorithm & Novel bat algorithm \\
\hline 0.5 & 334 & 329 & 322 \\
\hline 0.9 & 382 & 357 & 324 \\
\hline
\end{tabular}

Thus, the number of iterations of the novel bat algorithm is at least under the same conditions, which means that its time complexity reduce. In summary, the time complexity of novel bat algorithm is the most optimal.

\section{Conclusions}

Novel bat algorithm has been validated in the test and achieved good results. Novel bat algorithm is a more practical method, convergence is fast, and time distributes uniform. ourse scheduling problem is a multidisciplinary hard problems, this article attempt the novel bat algorithm only on the field of course scheduling, the effect on the algorithm need be improved in further.

\section{Acknowledgment}

This work is supported by Gansu Provincial College graduate tutor of scientific research project (GSGXGO30) and Dean Fund of Gansu Normal College for Nationalities (15-07).

\section{References}

[1] M.Dimopoulou, P.Miliotis.An automated university course timetabling system developed in a distributed environment: A case study[J]. European Journal of Operational Researeh. 2004: 136-147.

[2] ShawChingCharlga, YuChiChung. From timetslbling to train regulation-a new train operation model[J]. Information and Software Technology, 2005(47):575-585.

[3] Eduardo Santana de Almeida, Alexandre Alvaro, et a.lComponent Reuse in Software Engineering[M].C.E.S.A.R e-books, 2007.

[4] Garey M R, Johnson D S. Computers and Intractability: A Guide to the Theory of NP-Completeness[M]. San Franciso,CA: Freeman,4,109-122(1979).

[5] Holland J H. Genetic Algorithms and the Optimal Allocation of Trials [J]. SIA M J Computer, 1973: 2 (2): 89-104.

[6] Xinshe Yang. A new meta heuristic bat-inspired Algorithm [J], Nature Inspired Cooperative Strategies for Optimization (NICSO2010)(Eds. J. R. Gonzalez et al.), SCI 284, 2010:65-74.

[7] Xinshe Yang. Bat algorithm for multi-objective optimization [J]. Bio-Inspired Computation, 2011,3(5):267-274. 